The Astrophysical Journal, 621:1146-1152, 2005 March 10

(C) 2005. The American Astronomical Society. All rights reserved. Printed in U.S.A.

\title{
LEONID METEOROID ORBITS PERTURBED BY COLLISIONS WITH INTERPLANETARY DUST
}

\author{
Joser M. Trigo-Rodríguez, ${ }^{1}$ Hans Betlem, ${ }^{2}$ and Esko Lyytinen ${ }^{3}$ \\ Received 2004 September 16; accepted 2004 November 18
}

\begin{abstract}
By analyzing high-accuracy Leonid orbits obtained from multiple-station meteor observations during the 1998 outburst and 1999 storm, we have detected the presence of meteoroids with peculiar orbits. These meteoroids are characterized by having a geocentric radiant nearly identical to the members of the storm that appeared with them but showing different orbital elements owing to their lower geocentric velocities. The changes in some orbital elements are significant and allow us to interpret the cause of such differences. Mainly, the semimajor axis and the eccentricity of these anomalous orbits are lower than expected for members of the dust trail or members coming from the background component linked to the annual stream. From these characteristics it is likely that these peculiar meteoroids suffered some perturbations on very short timescales. We have investigated several causes, including planetary perturbations, collisions, and radiative effects in order to explain the observed orbital changes. We conclude that these orbital changes can be explained by the loss of orbital energy close to the ecliptic plane. Such an effect can only be explained clearly by collisions of the original meteoroids with dust particles associated with the zodiacal dust cloud. We have applied this hypothesis in order to constrain some physical properties of 55P/ Tempel-Tuttle cometary meteoroids.
\end{abstract}

Subject heading: meteors, meteoroids — comets: individual (55P/Tempel-Tuttle)

\section{INTRODUCTION}

Precise orbits and trajectories of meteors provide insight into the orbital dynamics of meteoroids, the processes that occurred to them in the interplanetary medium, and their physical properties. Ceplecha et al. (1998) and Jenniskens (1998) discussed the intrinsic interest in obtaining accurate trajectory data and meteoroid orbits from multiple-station meteor observations. Detailed campaigns during periods of high meteoric activity can provide sufficiently high numbers of orbits to find answers to several open questions. These questions relate to the physical properties of meteoroids, the processes that they suffer in the interplanetary medium, the role that these processes play in subsequent orbital evolution, and the decrease of the spatial density of the meteoroid streams. The study of cometary dust trails intercepted by the Earth can provide even more information because of the expected similarity in the orbits of the meteoroids (Jenniskens 1998). In fact, the young meteoroids belonging to a dust trail have very similar orbital parameters, characterized by a very small dispersion in the node of their orbits. As a consequence, the detailed study of the orbits of such meteoroids allows the identification of processes that would be unnoticed in older, and consequently more perturbed, members of the annual stream.

Dust trails released by the 55P/Tempel-Tuttle comet intercepted by the Earth periodically produce an important influx of cometary matter that reaches the atmosphere (Kresak 1993). The gradual decrease in rates during subsequent Leonid outbursts can be explained by the dispersion in the plane of the comet orbit, but the thickness of the trail producing different storms is apparently invariant (Jenniskens 1998). The influence

\footnotetext{
1 Astrobiology Center, Institute of Geophysics and Planetary Physics, University of California, Los Angeles, CA 90095-1567; jtrigor@ucla.edu.

2 Dutch Meteor Society, Lederkarper 4, 2318 NB Leiden, Netherlands; betlem@strw.leidenuniv.nl.

${ }^{3}$ Kehäkukantie 3 B, 00720 Helsinki, Finland; esko.lyytinen@jippii.fi.
}

of the combined gravitational field of the planets has been demonstrated to be important in some parts of the trails ( $\mathrm{Wu}$ $\&$ Williams 1996). In any case, other mechanisms can significantly reduce the spatial number density of particles belonging to a cometary dust trail. In this paper we refer to large Leonid particles producing the brightest Leonid meteors. The particles that we analyze here are in the mass range of several grams down to $4 \mathrm{mg}$; these produce Leonid meteors of negative stellar magnitude. Small particles are evolving under the main influence of the Poynting-Robertson drag, having negligible crosssectional areas and, consequently, a small probability of collision with other solar system particles. However, the large particles analyzed in our sample are probably destroyed by collisions before their semimajor axes change noticeably because of the Poynting-Robertson effect. In consequence, our sample contains meteoroids with a limited mass range, with typical lifetimes constrained by collisions with interplanetary dust. The meteoroids reported here appeared simultaneously with Leonid outbursts or storms during the last returns of 55P/Tempel-Tuttle to perihelion showing similar geocentric radiants but anomalous orbits. In consequence, since we can infer the release ages of particles belonging to a dust trail (Asher 1999; Betlem et al. 1999; TrigoRodríguez et al. 2004) our observations are important in deducing the significance of the collisional process in such dust structures. Nearly all reported Leonid filaments producing Leonid outbursts are only a few centuries old (McNaught \& Asher 1999, 2001, 2002); consequently, the detection of an orbital signature of impact processes on such particles on such short timescales is remarkable.

Until now the collisional destruction of finite-sized objects has been the subject of experimental and theoretical studies aimed at a better understanding of the evolution of the interplanetary meteoroid population. Unfortunately, it is difficult to obtain direct evidence of these processes of degradation in interplanetary meteoroids because this requires the study of small particles whose orbits usually evolve very quickly. The main goal of this paper is to give the first evidence of these processes 
and demonstrate that the interaction with interplanetary dust can be detected by obtaining high-precision orbits of meteoroids belonging to a stream and by looking for anomalous meteors. The detection of these processes has been made possible because in the last decade several teams of professional and amateur astronomers have obtained hundreds of valuable multiplestation photographs during Leonid storms. The teams, looking for the best sky conditions and equipped with batteries of cameras, have been capable of obtaining high-resolution images of the meteors. In addition, analysis of the multiple-station images of meteors using ever more sophisticated software for astrometric reduction has provided tens of accurate meteor orbits that a few decades ago would have required many years to be collected. Our results prove that these orbital data can provide significant advances in our knowledge of the evolution of meteoroid streams. On the basis of programmed multiple-station work, the Dutch Meteor Society (DMS) and the Spanish Photographic Meteor Network (SPMN) achieved fruitful small-camera network campaigns (Betlem et al. 1997, 1999, 2000; Trigo-Rodríguez et al. $2002,2004)$. In our previous work we obtained a high number of accurate Leonid orbits, which provide us with the opportunity to study statistically rare processes that would otherwise have been difficult to observe.

It is commonly assumed that cometary meteoroids have porous structures characterized by material of special fragility. Verniani (1969) revisited this topic and examined photographic and radio meteor data. He concluded that most meteoroids of cometary origin are porous, crumbly objects composed of conglomerate, sponge-like material. The fluffy nature of cometary meteoroids is also evident in the study of interplanetary dust particles (IDPs) that likely originate in short period comets (Rietmeijer 2002a, 2002b). In fact, the mineralogy and textures of chondritic aggregate IDPs are different from those of other materials in meteorite collections (Mackinnon \& Rietmeijer 1987). In this paper we present evidence of the presence in the dust trails of 55P/Tempel-Tuttle of centimeter-sized Leonid meteoroids with peculiar orbital differences; we demonstrate that the most likely explanation for these particles is that they collide with interplanetary dust particles. This discovery suggests that collisional processes can play an important role in the progressive decrease in the density of the 55P/Tempel-Tuttle dust trails, even on timescales of a few centuries.

\section{OBSERVATIONS AND DATA REDUCTION}

The observational procedures are explained in detail in previous papers, where the different observing sites are also given (Betlem et al. 1997, 1999, 2000; Trigo-Rodríguez et al. 2002). The observations were made by using batteries of cameras equipped with rotating shutters capable of measuring with precision the velocity of the meteors. Small batteries of cameras give observers the autonomy to establish campaigns in remote places in order to observe in excellent sky conditions. The DMS and SPMN teams made a special effort to obtain high-resolution meteor images during the Leonid storms that occurred between 1995 and 2002. Both teams followed the criterion of placing the stations in a north-south orientation in order to obtain images of meteors in the best geometry for triangulation because the Leonid meteors move predominantly from east to west during the night. As a consequence of the long meteor paths exhibited in such particular geometry, we can minimize the observational errors in astrometric and velocity measurements. Data reduction was done using similar analytical procedures that lead to orbits of high accuracy. Betlem et al. (1999) and Trigo-Rodríguez et al. (2002) provided more details about the reduction procedures for the DMS and SPMN orbital databases.

The importance of following a rigorous method of observation and reduction of the images is a key point in determining accurate meteoroid orbits. We have taken into account the observational and reduction uncertainties in order to give errors in the estimated trajectory, radiant data, and orbital parameters. These errors allow us to discard orbits affected by observational or reductional biases. Unfortunately, in the past orbital databases did not include information on the error in the orbital elements. As a consequence, all these meteor data are more difficult to interpret with confidence; there is great uncertainty in determining the true cause of anomalous orbits in such cases.

We have searched in the DMS and the SPMN databases for the presence of Leonid orbits with peculiar short semimajor axes. The reliability of these orbits with short semimajor axes was first noticed in a very accurate sample of the 1998 Leonid outburst (Betlem et al. 1999), where 3 of 98 analyzed meteoroid orbits had a shorter semimajor axis than expected. In the following Leonid return, an intense storm occurred, which was observed from Spain by DMS and SPMN teams (Betlem et al. 2000; Trigo-Rodríguez et al. 2002). It was in this 1999 Leonid return that, inside a sample of 18 Leonid orbits, we detected other peculiar short semimajor axis Leonid orbits (Trigo-Rodríguez et al. 2002). Two additional anomalous Leonid meteors were photographed in subsequent campaigns, one in 2000 and the other in 2001. These six orbits are listed in Table 1. The scattering in these anomalous short semimajor axis Leonid orbits suggests a random process that induces loss of orbital energy (Fig. 1). The origin of such meteoroids was an intriguing puzzle that induced us to check the presence of additional short semimajor axis Leonids in the literature. In order to find more cases we checked the IAU photographic database (Lindblad 1987, 1991). As a result we found three additional Leonid orbits, which have been included in Table 2. The quality of some of these older IAU orbits (especially $05501219 \mathrm{M}$ ) requires a case-by-case discussion because the uncertainties in the computation of the orbital elements were not given. In any case, we do not dedicate additional effort to this because the possibility exists that some of the orbits were affected by mistakes in the observational or reduction procedures. In consequence, we principally discuss in this paper data in which the uncertainties are reported.

\section{THEORY TO ANSWER THE ORIGIN OF THE SHORT-PERIOD LEONIDS}

\subsection{Use of the Tisserand's Criterion}

In this section we investigate whether planetary perturbations can cause a shortening of the orbital period. In order to do this we have used the Tisserand's criterion, assuming that the perturbing planet has a circular orbit. In this particular case the Tisserand's criterion can be written in the form

$$
\frac{1}{a}+2 \cos i \sqrt{a\left(1-e^{2}\right)}=C
$$

where $C$ is a constant for the solar system body in question and $a, e$, and $i$ are the semimajor axis, eccentricity, and inclination of the object's orbit, respectively. In this approach, the orbital element $i$ is relative to the perturbing planet's orbital plane and the semimajor axis of the perturbing planet has been chosen as unity; we express the semimajor axis of the comet or meteoroid in question as $a$. 
TABLE 1

Leonids AnALYZed Here

\begin{tabular}{|c|c|c|c|c|c|c|c|c|c|c|c|c|}
\hline \multirow[b]{2}{*}{ Code and Date } & \multicolumn{2}{|c|}{ Geocentric Radiant } & \multirow[b]{2}{*}{$M$} & \multirow[b]{2}{*}{$\begin{array}{c}H_{b} \\
(\mathrm{~km})\end{array}$} & \multirow[b]{2}{*}{$\begin{array}{l}H_{\text {end }} \\
(\mathrm{km})\end{array}$} & \multirow[b]{2}{*}{$\begin{array}{c}V_{g} \\
\left(\mathrm{~km} \mathrm{~s}^{-1}\right)\end{array}$} & \multirow[b]{2}{*}{$\begin{array}{c}q \\
(\mathrm{AU})\end{array}$} & \multirow[b]{2}{*}{$\begin{array}{c}1 / a \\
\left(\mathrm{AU}^{-1}\right)\end{array}$} & \multirow[b]{2}{*}{$e$} & \multirow[b]{2}{*}{$\begin{array}{c}i \\
(\operatorname{deg})\end{array}$} & \multirow[b]{2}{*}{$\begin{array}{c}w \\
(\mathrm{deg})\end{array}$} & \multirow[b]{2}{*}{$\begin{array}{c}\Omega \\
(\operatorname{deg})\end{array}$} \\
\hline & $\begin{array}{l}\text { R.A. } \\
\text { (deg) }\end{array}$ & $\begin{array}{l}\text { Decl. } \\
\text { (deg) }\end{array}$ & & & & & & & & & & \\
\hline 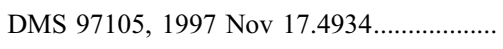 & 154.1 & +22.2 & -4 & 106.0 & 81.6 & $70 \pm 1$ & $0.9825 \pm 0.0009$ & $0.24 \pm 0.09$ & $0.77 \pm 0.7$ & $161.16 \pm 0.33$ & $170.3 \pm 0.9$ & 235.20 \\
\hline  & 152.2 & +22.0 & -1 & 99.2 & 78.2 & $60.8 \pm 0.4$ & $0.9722 \pm 0.004$ & $0.89 \pm 0.03$ & $0.13 \pm 0.03$ & $160.65 \pm 0.12$ & $145 \pm 6$ & 234.17 \\
\hline DMS 98020, 1998 Nov $16.854 \ldots \ldots \ldots \ldots \ldots \ldots \ldots$ & 153.2 & +21.8 & -4 & 122.8 & 88.5 & $69.4 \pm 0.3$ & $0.9814 \pm 0.0002$ & $0.21 \pm 0.03$ & $0.79 \pm 0.03$ & $162.36 \pm 0.06$ & $169.4 \pm 0.3$ & 234.25 \\
\hline 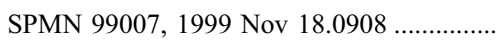 & 155.1 & +21.6 & -4 & 98.8 & 87.7 & $65.7 \pm 0.2$ & $0.9715 \pm 0.0005$ & $0.51 \pm 0.19$ & $0.503 \pm 0.013$ & $160.79 \pm 0.07$ & $161.5 \pm 0.04$ & 235.26 \\
\hline 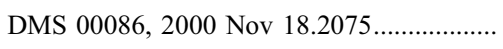 & 154.5 & +22.1 & -5 & 116.9 & 86.5 & $68.8 \pm 0.1$ & $0.9849 \pm 0.0007$ & $0.254 \pm 0.07$ & $0.750 \pm 0.007$ & $161.1 \pm 0.3$ & $172.6 \pm 0.7$ & 236.15 \\
\hline DMS 01390, 2001 Nov $18.5315 \ldots \ldots \ldots \ldots \ldots \ldots . . . . .$. & 153.8 & +21.8 & -2 & 128.5 & 88.5 & $68.3 \pm 0.1$ & $0.9867 \pm 0.0001$ & $0.308 \pm 0.007$ & $0.697 \pm 0.007$ & $161.82 \pm 0.06$ & $174.71 \pm 0.15$ & 236.23 \\
\hline 1998 Leonids $(N=76)$ & 153.3 & +22.1 & $\ldots$ & $\ldots$ & $\ldots$ & $70.5 \pm 0.4$ & $0.9839 \pm 0.0014$ & $0.10 \pm 0.03$ & $0.89 \pm 0.09$ & $162.05 \pm 0.49$ & $171.9 \pm 1.5$ & $\ldots$ \\
\hline 1999 Leonids $(N=116)$ & 153.4 & +21.8 & $\ldots$ & $\cdots$ & $\ldots$ & $70.6 \pm 0.3$ & $0.9867 \pm 0.0001$ & $0.10 \pm 0.02$ & $0.90 \pm 0.03$ & $162.50 \pm 0.22$ & $172.4 \pm 0.9$ & $\ldots$ \\
\hline 2000 Leonids $(N=71)$ & 154.2 & +21.6 & $\ldots$ & $\cdots$ & . & $70.5 \pm 0.4$ & $0.985 \pm 0.001$ & $0.11 \pm 0.03$ & $0.90 \pm 0.03$ & $162.40 \pm 0.6$ & $173.5 \pm 1.2$ & $\ldots$ \\
\hline 2001 Leonids $(N=262)$ & 154.2 & +21.6 & $\ldots$ & $\ldots$ & $\ldots$ & $70.7 \pm 0.3$ & $0.985 \pm 0.001$ & $0.10 \pm 0.06$ & $0.91 \pm 0.03$ & $162.40 \pm 0.6$ & $173.5 \pm 1.4$ & $\ldots$ \\
\hline 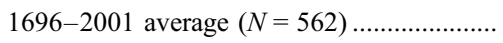 & 153.9 & +21.7 & $\ldots$ & $\ldots$ & $\ldots$ & $70.6 \pm 0.6$ & $0.984 \pm 0.006$ & $0.10 \pm 0.06$ & $0.90 \pm 0.03$ & $162.3 \pm 0.7$ & $173.0 \pm 2.9$ & $\ldots$ \\
\hline
\end{tabular}

Notes.-Meteor code and date of observation, radiant data, panchromatic magnitude $(M)$, beginning and ending heights, geocentric velocity, and orbital parameters of the Leonids analyzed here. The first two digits of the

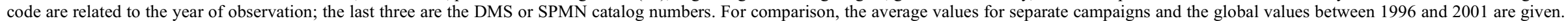




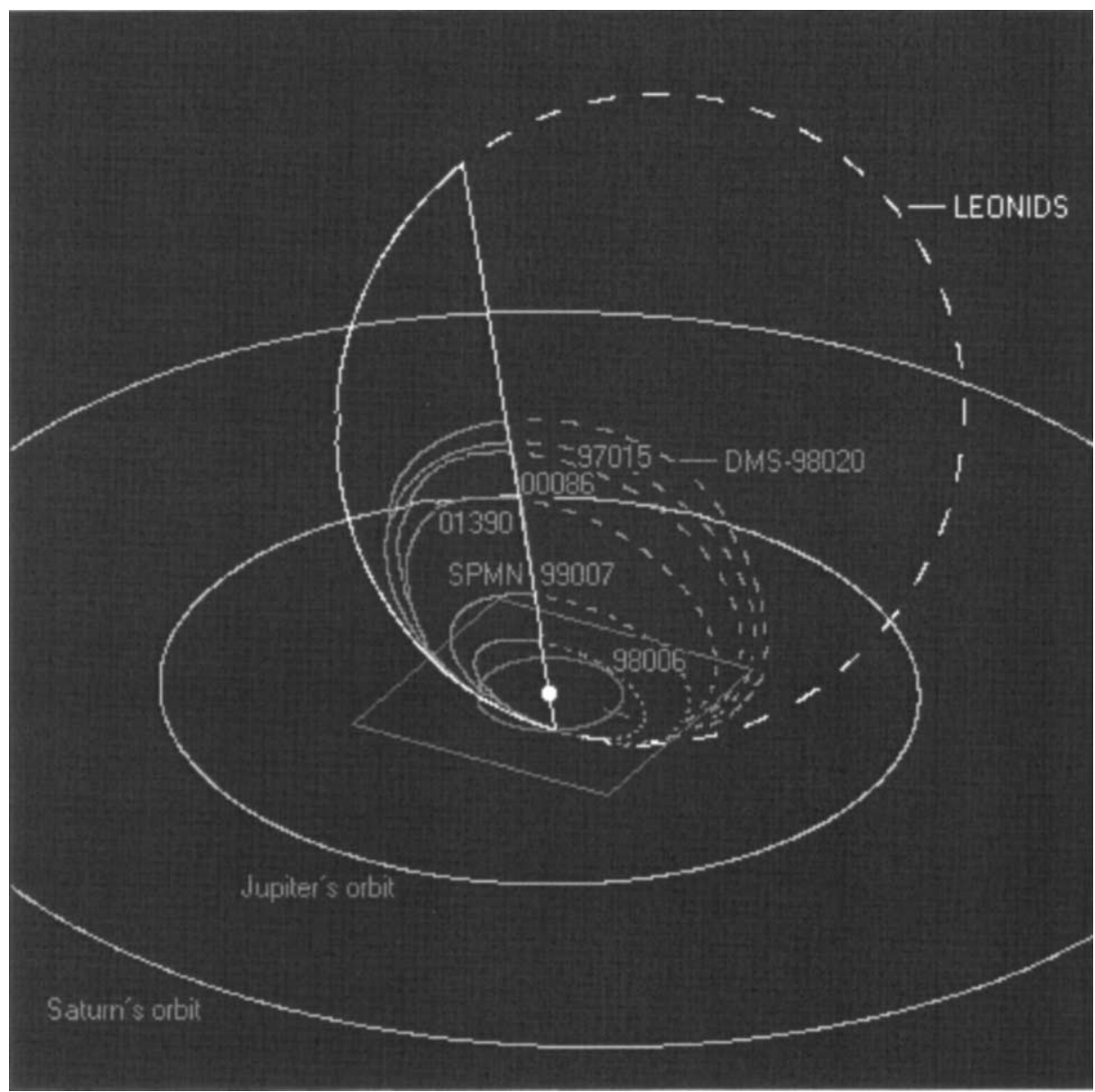

Fig. 1.-Comparison between the typical Leonid orbit and the anomalous orbits studied in Table 1. The Earth's orbit and the corresponding ecliptic plane are plotted in gray. For more details see the text.

For our purposes we modify the previous equation by writing $a\left(1-e^{2}\right)$ as $q(1+e)$ and moving $1 / a$ to the other side of equality,

$$
2 \cos i \sqrt{q(1+e)}=-\frac{1}{a}+C
$$

Equation (2) is suitable for our purposes because the Leonids have been observed near perihelion and because, for the sample of perturbed Leonids, the distance to perihelia $(q)$ has practically not changed from the value observed for the components of their respective dust trails.

The procedure consists in studying how the left-hand side of equation (2) has changed in the short-period Leonids compared to the parent comet or the "normal" members of the dust trail.
Because the orbit is retrograde, we have to keep in mind that $\cos i$ is negative. In the left-hand side, the eccentricity $e$ gets smaller when the orbital period is shorter because $q$ was almost fixed. Thus the value of the square root clearly becomes smaller. Since the Leonid stream has an inclination not very far from $180^{\circ}$, the changes that happen as a consequence of a change in inclination $i$ seem to be of lesser effect. Actually, the $i$ has become smaller, i.e., the orbit becomes more normal to the planet orbit, thus making the absolute value of $\cos i$ smaller. The change of both $e$ and $i$ seems to decrease the absolute value of the lefthand side. The real value increases.

Note that in the right-hand side of equation (2), 1/a increases when a gets smaller. This means that $-1 / a$ decreases, which is in the opposite direction of the left-hand side change expected

TABLE 2

Additional Cases of Short-Period Leonid Orbits

\begin{tabular}{|c|c|c|c|c|c|c|c|c|c|c|c|c|c|}
\hline IAU CODE & \multicolumn{2}{|c|}{ GEocentric Radiant } & $\begin{array}{l}\text { DATE (Nov) } \\
\text { (Day Year) }\end{array}$ & MAGNITUDE & $\begin{array}{c}H_{b} \\
(\mathrm{~km})\end{array}$ & $\begin{array}{l}H_{\text {end }} \\
(\mathrm{km})\end{array}$ & $\begin{array}{c}V_{\mathrm{g}} \\
\left(\mathrm{km} \mathrm{s}^{-1}\right)\end{array}$ & $\begin{array}{c}q \\
(\mathrm{AU})\end{array}$ & $\begin{array}{c}1 / a \\
\left(\mathrm{AU}^{-1}\right)\end{array}$ & $e$ & $\begin{array}{c}i \\
(\operatorname{deg})\end{array}$ & $\begin{array}{c}w \\
(\mathrm{deg})\end{array}$ & $\begin{array}{c}\Omega \\
(\operatorname{deg})\end{array}$ \\
\hline 01898099W..... & 153.2 & +21.8 & 17.3441949 & -1.8 & 102.2 & 92.8 & 62.3 & 0.962 & 0.763 & 0.265 & 157.8 & 150.6 & 234.7 \\
\hline 05501219M...... & 157 & +20 & 20.51952 & -0.8 & 113.4 & $\ldots$ & 69.3 & 0.98 & 0.227 & 0.78 & 163 & 169 & 238 \\
\hline 08446534D...... & 152.4 & +22.4 & 17.9531958 & -6.1 & 108 & 90 & 62.1 & 0.985 & 0.806 & 0.209 & 160.2 & 193 & 235 \\
\hline
\end{tabular}

Notes.-Additional cases of short-period Leonid orbits from the International Astronomical Union Database (Lindblad 1987, 1991). Orbital changes are similar to those reported for our Leonid data. 
TABLE 3

Apparent Radius of Leonid Meteoroids

\begin{tabular}{|c|c|c|c|c|}
\hline \multirow[b]{2}{*}{ MAGNITUde } & \multirow{2}{*}{$\begin{array}{c}\text { MASS } \\
(\mathrm{mg})\end{array}$} & \multicolumn{3}{|c|}{ RADIUS (mm) } \\
\hline & & For $\delta=0.5 \mathrm{~g} \mathrm{~cm}^{-3}$ & For $\delta=1 \mathrm{~g} \mathrm{~cm}^{-3}$ & For $\delta=2 \mathrm{~g} \mathrm{~cm}^{-3}$ \\
\hline+1 . & 0.6 & 0.7 & 0.5 & 0.4 \\
\hline 0 & 1.5 & 0.9 & 0.7 & 0.6 \\
\hline-1 & 4.1 & 1.3 & 1.0 & 0.8 \\
\hline-2 & 11 & 1.7 & 1.5 & 1.1 \\
\hline-3 & 30 & 2.4 & 1.9 & 1.5 \\
\hline-4 & 82 & 3.4 & 2.7 & 2.1 \\
\hline-5 & 224 & 4.7 & 3.8 & 3.0 \\
\hline
\end{tabular}

Notes.-Apparent radius of Leonid meteoroids assuming spherical shapes and typical proposed densities. Mass of the particles was derived from the Verniani (1973) equation using an average geocentric velocity of $72 \mathrm{~km} \mathrm{~s}^{-1}$.

from the short-period Leonids presented in Table 1. As was mentioned previously, the unity in the criterion is the semimajor axis of the perturbing planet and we did not pay attention to this in the changes in the different sides of equation (2). Whether the planet under consideration is Jupiter or Saturn, the treatment of the direction of the changes contemplated here is fully valid.

A more detailed study, however, reveals the following. The left-hand side of equation (2) is proportional to the orbital angular momentum of the Leonid meteoroid in question, relative to the perturbing planet orbital revolution axis, and $-1 / a$ is proportional to the meteoroid's orbital energy. The criterion relates the mutual changes of these parameters. We can compare the orbital changes caused, for example, by Jupiter and by Saturn. In the perturbations caused by both planets the change in the angular momentum compared to the change in $a$ is smaller for Jupiter (the inner planet) than for Saturn. It is theoretically possible that Saturn could have lengthened the orbital period of the meteoroid and Jupiter could have shortened it by an amount larger than the final direction of the left-hand side of (2). In such a case, the result would be to the other direction than would follow when we study these as separate. However, the apparent changes in both sides of equation (2) are so large that they require large changes to both directions in the orbital period induced by perturbations of planets in several highly perturbing instances. Consequently, we conclude that the reduction of the orbital period of Leonid meteoroids belonging to a young dust trail is improbable in our cases. Such a scenario is even more improbable because of the young nature of the studied trails and the necessity of explaining how the process could be "selective" by affecting only a small sample of the observed meteoroids.

\subsection{Agreement between Observations and Collisional Theory}

Collisions between meteoroids can be catastrophic, causing the volatilization of both particles, or producing erosion of the bigger one during the impact and volatilization of the impactor. Whether a particular collision will be catastrophic or erosive depends on several parameters, i.e., the mass, density, and compressive strength of the target; the mass of the projectile; and the relative velocity of the particles. Grün et al. (1985) included some of the physical parameters into the so-called $\Gamma$-factor $\left(\Gamma_{0}\right)$. According to the theory described by these authors, the condition of catastrophic disruption of a target particle with mass $\left(m_{1}\right)$ is
The target will survive when its mass is greater than the mass of the projectile $\left(M_{2}\right)$ times the $\Gamma$-factor:

$$
m_{1}>\Gamma_{0} m_{2}
$$

In both equations the $\Gamma$-factor appears, which can be written

$$
\Gamma_{0}=9.76 \times 10^{2} S_{c}^{-0.45}\left(\frac{m_{1}}{\rho_{1}}\right)^{0.075} V^{2} .
$$

In the previous equation, there appeared the unconfined compressive strength of the material $\left(S_{c}\right)$ given in kbar, the mass and density of the target $\left(m_{1}\right.$ and $\left.\rho_{1}\right)$ in units of $\mathrm{g}$ and $\mathrm{g} \mathrm{cm}^{-3}$, respectively, and the encounter relative velocity $(V)$ given in $\mathrm{km} \mathrm{s}^{-1}$. In general, a catastrophic collision will occur if the mass of the projectile is less than or equal to a constant times the mass of the target.

Equation (3) provides the possibility of testing some of our ideas. From photographic data we have obtained all the cases that are associated with particles that produce meteors of magnitude at least -1 . If we assume that the observed cases are the remnants of a collision between an interplanetary dust particle and a relatively large Leonid meteoroid, we can conclude that the failure of detecting perturbed orbits for the smaller particles is due to Leonid particles having suffered catastrophic collisions. In fact, equation (3) suggests that the smallest Leonid meteoroids have fewer possibilities to survive impacts with zodiacal dust. In order to check if such a hypothesis is reasonable, we consider a "typical collision" under different assumptions. First we assume the maximum mass for zodiacal dust particles to be $10^{-6} \mathrm{~g}$ because masses greater than this are quickly destroyed by mutual collisions (Grün et al. 1985, 2002) while the mass of a meteoroid producing a zero-magnitude Leonid meteor is $\approx 2 \mathrm{mg}$ (Table 3 ). Applying both values to equation (3), we impose the equality condition as an extreme case of disruption due to the observational absence of short-period Leonid orbits with lower masses. From the equality of such an equation and the mass of the involved particles we calculated the $\Gamma$-factor to be $\Gamma \sim 4000$. To take into account the presumably porous nature of the target, we assume in the calculation that the density of the target is around $1 \mathrm{~g} \mathrm{~cm}^{-3}$. In order to estimate the relative velocity of the encounter we can use the equation

$$
V^{2}=V_{1}^{2}+V_{2}^{2}-2 V^{1} V_{2} \cos \phi
$$


where $\phi$ is the encounter angle and $V_{1}$ and $V_{2}$ are the velocities of the impacting meteoroids. From this equation, assuming that the encounter happened close to the ecliptic plane, we can approximate that the encounter angle is given directly by the inclination of the Leonid meteoroid stream $\left(\phi=162^{\circ}\right)$. Taking this angle, the average heliocentric velocity for zodiacal dust of $15 \mathrm{~km} \mathrm{~s}^{-1}$ (Grün et al. 1985), and the typical heliocentric velocity for Leonid meteoroids of $41 \mathrm{~km} \mathrm{~s}^{-1}$, we can obtain the relative velocity of a "typical" collision between both sources. The presence of prograde zodiacal dust can induce a collision at a relative velocity of $55 \mathrm{~km} \mathrm{~s}^{-1}$. We consider that collision with retrograde dust is unlikely owing to the low survival timescale of zodiacal dust moving in such a direction.

With this assumption from equation (5), it is possible to obtain the unconfined compressive strength for these particles: $2.8 \mathrm{kbar}$ for the collision with prograde dust. This value is in the range reported by Grün et al. (1985). Although the model is simple and involves several assumptions, its application gives coherent results and can provide new insights about the collisions between fluffy cometary particles and small mineral grains at high velocities. In fact, until now experimental techniques in the laboratory have been able to reach only lower velocities: $V \leq$ $10 \mathrm{~km} \mathrm{~s}^{-1}$ (Nikolova \& Jones 2001).

\section{DISCUSSION}

Observational results frequently indicate that cometary particles appear fragile (Verniani 1969; Fomenkova et al. 1994). Their lifetimes are usually inferred from theoretical or experimental approaches including several not very realistic assumptions. For example, the measurement of the space exposure times inferred from the measured abundances of ${ }^{26} \mathrm{Al}$ and ${ }^{10} \mathrm{Be}$ in cosmic spherules (Nishiizumi et al. 1991) are as much as 2 orders of magnitude greater than the collisional lifetimes predicted on the basis of laboratory impact results. Love et al. (1993) proposed that such differences can be attributed to differences in the physical properties of the experimental targets compared to real meteoroids.

The reported survival of cometary meteoroids from encounters with relatively high-velocity impactors provides evidence that porosity can play an important role in collisional processes involving these particles. The idea that aggregate fractal shapes are typical for fluffy cometary meteoroids is supported by observations of IDPs of presumably cometary origin (Rietmeijer 2002a, 2002b) and by analyses of 1P/Halley dust (Fomenkova et al. 1994). According to the experimental simulations made by Love et al. (1993) for porous targets, the kinetic energy of the projectile is partitioned into target heating and into the spallation, disruption, and crushing of the component grains to fill the void spaces opened in the meteoroid. In such a case, the survival of large meteoroids under a particular impact geometry and low-mass impactors seems feasible.

The fact that the anomalous orbits reported here are associated with large meteoroids suggests that the smallest particles are not able to survive such collisional processes. In particular, the absence of perturbed orbits for meteoroids producing meteors under magnitude -1 suggests that this would be the minimum photometric magnitude reached by meteoroids able to survive a catastrophic fragmentation. From the estimated radius for Leonid meteoroids of assumed spherical shape and with the typical densities of IDPs reported by Rietmeijer (2002c) in Table 3, we suggest that catastrophic fragmentation would be characteristic of submillimeter particles with a radius below $1 \mathrm{~mm}$.

According to the proposed scenario, particles smaller than $\sim 1 \mathrm{~mm}$ (Table 3 ) would be completely disrupted in such im- pacts and thus would not be observed. In any case, the sample of perturbed meteoroids reported here is small; future confirmation and theoretical work would be of great interest.

The different ablation heights (Table 1) for the peculiar Leonid meteoroids are a direct consequence of the decrease in the geocentric velocity of the particles during atmospheric interaction. We cannot discount the possibility that some particles would reach deep parts of the atmosphere as a consequence of the increase in the material strength produced by compaction during the impact. This would be analogous to the simulations of Love et al. (1993), but again this requires more observational data.

From orbital mechanics, the most probable place for a collision to occur is where the Leonid meteoroid crosses the ecliptic plane. This fact links the collision with micrometeoroids belonging to the zodiacal dust cloud. Because of the particular highinclination orbit of the Leonid stream, collisions occurring farther from the Sun than at the Earth's orbital distance will change the $q$-value noticeably owing to the reduction in the heliocentric velocity. On the other hand, a typical collision near Earth's orbit would only minimally affect the $q$-value because the collision would occur not far from perihelion. This fact is clearly observed in Table 1. Reduction in velocity also affects the orbital period. In the vicinity of the Earth's orbit exists a large population of small particles moving near the ecliptic plane in the forward direction (i.e., prograde), so a collision with such a particle is highly probable.

The collision would reduce the orbital speed of the meteoroid in question, producing a shorter period meteoroid orbit as has been observed in our sample. If the colliding micrometeoroid moved in a prograde orbit near the ecliptic plane, this would reduce the absolute value of the left-hand side of equation (2). Practically any collision near the perihelion of the meteoroid orbit would result in the reduction of the square root value that appears on the left-hand side of equation (2). We assume that the zodiacal dust particles have orbits of small inclination and, to simplify the discussion, set the inclination to zero. We also assume that the direction of motion is in the ecliptic plane. During a collision, the velocity (and the orbital angular momentum relative to the axis normal to the ecliptic plane) decreases, but the velocity component in the direction of the $Z$ axis remains the same. After the collision, the Leonid meteoroid assumes an orbit more normal to the ecliptic, e.g., with systematically smaller inclinations than the average of particles in the stream (as has been observed in our sample, e.g., Table 1). In consequence, the short-period Leonid meteoroids reported here can be explained as having been caused by collisions with micrometeoroids moving near the Earth's orbital plane. We emphasize that this does not mean that such collisions would not occur at great heliocentric distances, but particles colliding at such distances will have a much lower probability of moving close to the Earth's orbit.

Finally, we note that mutual collisions between meteoroids of the same stream would also happen. Such encounters can be due to the different relative velocities between meteoroids of the same dust trail or with background Leonids, i.e., belonging to the annual stream. In most instances, resulting meteoroids will not have an orbital period smaller than either of the colliding meteoroids. In the case of fragmentation produced by the impact, meteoroid pieces could assume shorter orbital periods. However, there would probably not be such a systematic orbital inclination shift to the original orbits as observed. It is fortunate that we were dealing with retrograde meteoroids. With prograde meteoroids, the Tisserand's criterion could not have distinguished 
the shortening of the orbital period caused by planetary perturbations from collisions with micrometeoroids. Actually, such collisions would probably be much less common and there might not exist many such shorter period meteoroids. According to our data, the population of such meteoroids would be less than $1 \%$.

\section{CONCLUSION}

The detection of cometary meteoroids coming from highly perturbed orbits but still linked to a particular stream can be achieved by multiple-station meteor observations. These observations are important because they allow us to test processes that happened in the interplanetary medium, such as collisions. Meteoroids producing Leonid storms have a very well defined origin (Asher 1999) and follow very similar orbits on a timescale of a few centuries (Betlem et al. 2000; Trigo-Rodríguez et al. 2002, 2004). According to the reported orbital data of these anomalous Leonid meteoroids we conclude the following:

1. Some large particles intercepted by the Earth in recent Leonid storms had lesser geocentric velocities than expected for members of the Leonid stream. Consequently, the corresponding orbits exhibited shorter semimajor axes and smaller eccentricities than those observed for Leonid members. Despite these differences, such anomalous meteoroids had geocentric radiants similar to those exhibited by the members of the Leonid dust trails, suggesting that such particles are relatively young. However, the observed orbital element differences make it impossible to clearly link such meteoroids with the dust trails.
2. The most likely explanation for the reported orbital behavior (mainly lower eccentricity and shorter semimajor axis) is that these particles had previously suffered a collision. Orbital dynamics suggest that it happened when these Leonid meteoroids crossed the ecliptic plane. This suggests that collisions of Leonid meteoroids with zodiacal dust are common.

3. The short-period Leonid meteoroids would contribute to the enhancement of the background of bright Leonid meteors observed during years far from the return of their parent body (Jenniskens 1998). A search into the IAU Meteor Database shows three cases of bright meteors linked by their geocentric radiant to the Leonid meteoroid stream that exhibited similar low geocentric velocities but appeared in years in which there was no interception of Leonid dust trails by the Earth.

4. The collision with interplanetary meteoroids is the most reasonable explanation of the origin of the anomalous Leonid orbits reported here. In any case, we suggest that additional modeling and experiments on collisions with fluffy materials can help determine if the proposed scenario is correct. In addition, new multiple-station campaigns can bring out more cases to test, providing new insights on the origin and relative abundance of these interesting short-period Leonids.

The authors are grateful for helpful discussions with Alan Rubin (UCLA) and Frans Rietmeijer (University of New Mexico). J. M. T.-R. is grateful to the Spanish State Secretary of Education and Universities for a postdoctoral grant.
Asher, D. 1999, MNRAS, 307, 919

Betlem, H., Jenniskens, P., Spurný, P., Van Leeuwen, G. D., Miskotte, K., Ter Kuile, C. R., Zarubin, P., \& Angelos, C. 2000, Earth Moon Planets, 82, 277

Betlem, H., et al. 1997, Planet. Space Sci., 45, 853 1999, Meteoritics Planet. Sci., 34, 979

Ceplecha, Z. K., Borovicka, J. I., Elford, W. G., Revelle, D. O., Hawkes, R. L., Porubcan, V., \& Simek, M. 1998, Space Sci. Rev., 84, 327

Fomenkova, M. N., Chang, S., \& Mukhin, L. M. 1994, Geochim. Cosmochim. Acta, 58, 4503

Grün, E., Dikarev, V., Krüger, H., \& Landgraf, M. 2002, in Meteors in the Earth's Atmosphere, ed. E. Murad \& I. P. Williams (Cambridge: Cambridge Univ. Press), 35

Grün, E., Zook, H. A., Fechtig, H., \& Giese, R. H. 1985, Icarus 62, 244

Jenniskens, P. 1998, Earth Planets Space, 50, 555

Kresak, L. 1993, A\&A, 279, 646

Lindblad, B. A. 1987, in Interplanetary Matter, ed. Z. Ceplecha and P. Pecina (Prague: AICAS), 201

- 1991, in IAU Colloq. 126, Origin and Evolution of Interplanetary Dust, ed. A. C. Levasseur-Regourd and H. Hasegawa (Dordrecht: Kluwer), 311

Love, S. G., Hörz, F., \& Brownlee, D. E. 1993, Icarus, 105, 216

\section{REFERENCES}

Mackinnon, I. D. R., \& Rietmeijer, F. J. M. 1987, Rev. Geophys. 25, 1527

McNaught, R., \& Asher, D. J. 1999, WGN, J. Int. Meteor. Org., 27, 85

- 2001, WGN, J. Int. Meteor. Org., 29, 156

2002, WGN, J. Int. Meteor. Org., 30, 132

Nikolova, S., \& Jones, J. 2001, in Proc. Meteoroids 2001 Conf., Lifetimes of Meteoroids in Interplanetary Space: the Effect of Erosive Collisions and Planetary Perturbations, ed. B. Warmbein (ESA SP-495; Noordwijk: ESA), 581

Nishiizumi, K., Arnold, J. R., Fink, D., Klein, J., \& Middleton, R. 1991, Earth Planet. Sci. Lett., 104, 315

Rietmeijer, F. J. M. 2002a, in Meteors in the Earth's Atmosphere, ed. E. Murad \& I. P. Williams (Cambridge: Cambridge Univ. Press), 215 2002b, Chem. Erde, 62, 1

2002c, Earth Moon Planets, 88, 35

Trigo-Rodríguez, J. M., Llorca, J., \& Fabregat, J. 2002, Earth Moon Planets, 91, 107

Trigo-Rodríguez, J. M., Llorca, J., Lyytinen, E., Ortiz, J. L., Sanchez-Caso, A.,

Pineda, C., \& Torrell, S. 2004, Icarus, 171, 219

Verniani F. 1969, Space Sci. Rev. 10, 230 1973, J. Geophys. Res., 78, 8429

Wu, Z., \& Williams, I. P. 1996, MNRAS, 280, 1210 